\title{
Effets d'une contrainte hydrique sur le flux pédonculaire massique et la croissance de la pêche
}

\author{
JG Huguet *, M Génard \\ avec la collaboration technique de R Laurent, B Lebœuf, P Rouet
}

INRA, unité de recherche en écophysiologie et horticulture, domaine Saint-Paul, 84914 Avignon cedex 9, France

(Reçu le 24 octobre 1994 ; accepté le 11 avril 1995)

\begin{abstract}
Résumé - L'objectif de cette étude était d'établir, pendant la dernière phase de croissance active de la pêche (Prunus persica (L) Batsch cv Dixired), l'influence des conditions d'alimentation hydrique sur la croissance des fruits. Des pêchers sont cultivés en pots, selon 2 régimes de disponibilité en eau (avec ou sans restriction) et les microvariations du diamètre des troncs ont été enregistrées pour servir d'indicateur de la contrainte. Pour chaque traitement, l'évolution du diamètre de quelques fruits a été suivie par micromorphométrie. Les diamètres de toutes les autres pêches ont été suivis par mesures manuelles. La transpiration propre des fruits a été évaluée sur des fruits cueillis, en mesurant soit leurs pertes de masse, soit les variations micromorphométriques de leur diamètre. Sur la période considérée (juin 1993) l'accroissement pondéral moyen des pêches du traitement en restriction d'eau a été réduit à $62 \%$ de celle du témoin. Le flux pédonculaire massique d'approvisionnement du fruit est défini, sur un intervalle de temps donné, comme la somme des valeurs de l'accroissement pondéral et des pertes pondérales par transpiration. Les pertes par respiration ont été quantitativement négligeables. La transpiration de la pêche a représenté, en cumulé sur la période d'étude : $70 \%$ du flux pédonculaire. Lorsque la disponibilité hydrique n'était pas limitante, le flux pédonculaire, calculé au pas horaire, variait en relation positive avec la demande climatique en eau ; il était minimal au lever du jour, maximal dans l'après-midi. Le flux journalier a augmenté au cours des quelques semaines qui précèdent la maturité : il est passé d'une fraction de gramme à près de $30 \mathrm{~g} / \mathrm{j}$ pour un fruit moyen. Lorsque la disponibilité hydrique était réduite, le flux pédonculaire variait en raison inverse de la demande climatique pendant la phase éclairée du nycthémère. Dans les conditions de cette expérience, le cumul des flux pédonculaires massiques d'une pêche sous contrainte a correspondu à $66 \%$ de celui d'une pêche témoin. Nous avons trouvé une relation linéaire très significative entre la diminution, par rapport au fruit témoin, du flux pécondulaire massique d'une pêche sous contrainte hydrique et l'augmentation, par rapport à l'arbre témoin, du maximum de contraction diurne du tronc de l'arbre sous stress.
\end{abstract}

alimentation en eau / croissance / fruit / Prunus persica = pêche / micromorphométrie / bilan de flux

Summary - Effect of water availability on the pedoncular flow and the growth of peach trees. The aim of this study was to determine the flows that account for fruit growth in the peach tree (Prunus persica (L) Batsch, cv Dixired) under different conditions of water availability. Young peach trees were grown in pots and were either watered continuously (control plants) or irrigated with reduced amounts of water (stressed plants). Variations in trunk diameter were recorded using LVDT (linear variable differential transformer) gauges and were used as an index of the water stress level. On each treatment the diameter variations were also recorded on some of the fruits with the same gauges. The fruits without continuous recording were measured with a digital caliper. The transpiration rate was found by regular weighing of picked fruits or by recording diameter variations using LVDT gauges. The average weight increase on

* Correspondance et tirés à part 
stressed fruits was $62 \%$ of the control. Massic pedoncular flow was defined as the fresh weight increase plus the transpiration. Respiration losses were quantitatively negligible. During the period under study, the peach transpiration was about $70 \%$ of the pedoncular flow. When the tree was not stressed, the pedoncular flow, which was computed hourly, was positively linked to the climatic demand for water. It was minimum in the early morning and maximum in the midafternoon. The daily pedoncular flow increased from less than $1 \mathrm{~g}$ to about $30 \mathrm{~g}$ during the last month before maturity. The pedoncular flow in the stressed tree was negatively linked to the climatic demand for water during the photoperiod. The cumulated massic pedoncular flow of a stressed peach was $66 \%$ of the control. Compared with the control, there was a highly significant linear relationship between the decrease of the massic pedoncular flow of the water-stressed peach and the increase in the maximum daily shrinkage of its trunk.

\section{water availability / fruit growth / Prunus persica / micromorphometry / flow balance}

\section{INTRODUCTION}

En France la consommation globale de pêches et nectarines est en baisse régulière depuis 5 ans, sans relation directe avec le volume de production ou le niveau des prix de détail. Les différents opérateurs s'accordent sur la mise en cause de la perte de qualité tout au long de la filière comme responsable de cette désaffection (Delgado, 1994). Pour augmenter leur compétitivité les arboriculteurs doivent trouver une meilleure adaptation des productions pour satisfaire aux exigences finales des consommateurs en matière de qualité. II a été démontré (Chalmers et al, 1983 ; $\mathrm{Li}$ et Huguet, 1989) qu'une restriction de l'irrigation pourrait permettre d'améliorer la qualité organoleptique des pêches dans les régions de production où l'irrigation reste l'indispensable compensation d'une pluviométrie estivale généralement insuffisante. Cependant, cette amélioration de la qualité organoleptique s'accompagne souvent d'une diminution du calibre ( $\mathrm{Li}$ et Huguet, 1989). Ainsi la restriction de l'irrigation a des effets contradictoires sur la valeur économique de la récolte. Pour proposer des applications pratiques, il faudrait pouvoir conseiller le moment et l'intensité de la restriction afin d'obtenir un compromis économique acceptable entre la perte de calibre et l'amélioration d'autres paramètres de la qualité. Le calibre du fruit est encore le premier critère de fixation des prix et, quand la disponibilité en eau n'est pas limitante, il existe une relation positive entre la vitesse de croissance et la qualité des fruits d'un même arbre (Génard et al, 1991). La raison de cette liaison est que les fruits qui grossissent le plus vite sont ceux qui sont avantagés par un meilleur approvisionnement en assimilats. II paraît alors indispensable d'analyser comment une restriction hydrique modifie cette relation en analysant d'abord son effet sur la croissance de la pêche. Le souci d'efficacité pratique impose de choisir la dernière phase de croissance active des pêches comme phase d'ap- plication des restrictions. En effet, c'est durant cette phase que la pêche acquiert près de $75 \%$ de son poids final au cours d'une période où les restrictions de l'alimentation en eau sont facilement mises en œuvre.

Le flux pédonculaire massique d'approvisionnement du fruit est défini, sur un intervalle de temps donné, comme la somme de l'accroissement pondéral et des pertes par transpiration et respiration. Pendant l'intervalle de temps considéré, ce flux pédonculaire correspond au bilan des transits phloémien et xylémien en direction du fruit. Un certain nombre d'auteurs ont déjà étudié l'importance de ces flux par rapport à la croissance des fruits. Ho et al (1987) ont calculé les flux d'eau et de matière sèche qui alimentent des tomates cultivées en solution nutritive, et démontré que la voie phloémique était prédominante, que la vitesse d'accroissement pondéral était décroissante pendant le mois précédant la maturité et que la transpiration ne dépassait pas $1 \mathrm{~g}$ par fruit et par jour. Lee et al (1989) et Johnson et al (1992) ont suivi, en continu, les variations de diamètre et de potentiel hydrique de tomates en pots sous serre et mis en évidence le rôle moteur du gradient de potentiel hydrique entre la tige et le fruit pour expliquer les variations de diamètre de celui-ci. Lee (1990) propose un modèle de croissance de la tomate dans lequel le flux massique d'entrée et la transpiration surfacique horaire sont considérés comme constants ; le taux d'accroissement pondéral diminue proportionnellement à l'augmentation de la surface du fruit ; il devient nul lorsque la transpiration équilibre le flux d'entrée. Bussières (1994) considère que l'entrée d'eau dans le fruit de tomate est ralentie par des résistances qui augmentent avec sa taille. Lang et Thorpe (1989) puis Lang (1990) déterminent respectivement sur baies de raisin et sur différentes variétés de pommes l'importance relative des différents flux, dont la transpiration, pour expliquer, en particulier, la variabilité de composition minérale des 
fruits. Chez la pêche, Nobel (1975) a montré que la transpiration du fruit est forte et Selles (1988) avance que la diminution de diamètre des pêches pendant la journée peut être expliquée par les pertes transpiratoires.

De nombreux travaux ont mis en évidence un effet négatif d'un stress hydrique sur la croissance de la pêche (Chalmers et al, 1983 ; Li et al, 1989). Cependant, à notre connaissance, l'analyse des différents flux dont résulte la croissance n'a pas été faite. Aussi, notre premier objectif a été de comparer les flux entrant (massique pédonculaire) et sortant (transpiratoire ou respiratoire), pour des fruits en situation de stress hydrique ou d'alimentation en eau non limitante, au cours de la phase finale de croissance active des fruits (phase III).

\section{MATÉRIEL ET MÉTHODES}

L'essai a été conduit en plein air sur un terrain du domaine du centre de recherches d'Avignon, situation représentative du climat du sud de la vallée du Rhône. Nous avons utilisé 5 pêchers Dixired (4 ans) cultivés en pots de 80 I avec un substrat de sable grossier et un système d'irrigation goutte à goutte. Le suivi de cet essai a été réalisé du 28 mai au 28 juin 1993, c'est-àdire pendant la dernière phase de croissance active des fruits (phase III). Jusqu'au 8 juin, les 5 arbres de l'essai ont été irrigués en continu et sans restriction pendant les $12 \mathrm{~h}$ de la phase diurne. Les 3 arbres (témoins) ont conservé ce régime pendant toute la durée de l'expérimentation. Les 2 arbres (stressés) ont subi, successivement, 2 types de restriction en eau. Le premier a consisté à couper complètement l'alimentation en eau des arbres stressés pendant 3 j ( 8 au 10 juin) ; le second a consisté à limiter l'irrigation à $3 \mathrm{~h}$ en début de matinée (16 au 28 juin). Les 2 périodes de stress ont été séparées par un retour au régime témoin (11 au 15 juin).

Deux arbres (1 témoin, 1 stressé) ont été équipés de capteurs micromorphométriques (capteur de déplacement rectiligne à induction différentielle, ENERTECSchlumberger CD 4112). Les capteurs micromorphométriques posés sur les troncs permettaient, à travers l'observation des maxima de contraction diurne, d'accéder à un indicateur du niveau de la contrainte (Selles, 1988 ; Huguet et al, 1992). Le maximum de contraction diurne (MCD) correspond à la perte maximale de diamètre observée au cours de la phase diurne d'un nycthémère, c'est-à-dire la différence entre le diamètre à l'aube et le diamètre minimal observé dans la journée. Chez le pêcher, il a été prouvé que les microvariations de diamètre du tronc étaient en étroite corrélation avec les variations du stock d'eau de l'arbre (Simonneau et al, 1993), variations dont dépendent fonctionnellement les variations du potentiel hydrique de l'arbre. Cette relation justifie le choix du MCD comme indicateur du niveau de contrainte. Cinq pêches par arbre (1 arbre témoin, 1 arbre stressé) étaient équipées de capteurs fixés par des carpomètres (Huguet, 1985). Un carpomètre supplémentaire permettait de suivre les variations de diamètre d'une pêche détachée mais fixée sur l'arbre dans une position analogue à celle des autres pêches. Le suivi des variations de diamètre des fruits par des capteurs de déplacement linéaire a été employé par plusieurs auteurs pour accéder au calcul des flux, sur tomate (Lee et al, 1989 ; Johnson et al, 1992), et sur pommes (Lang, 1990). L'acquisition des données micromorphométriques, au pas horaire, a été assurée par 2 centrales “PEPISTA 3000-COPA-Informatique». Tous les fruits non équipés de capteur (une quinzaine par arbre) ont fait l'objet d'un suivi manuel, par pied à coulisse numérique, des variations de diamètre (tous les 4 jours, 3 arbres témoins et 2 arbres stressés).

\section{Calcul des flux à l'échelle de la journée}

Le flux pédonculaire massique d'entrée de la sève $(F P)$ est égal à la somme de l'accroissement pondéral du fruit $(A P)$, des pertes par la transpiration $(T r)$ et par les respirations de croissance $(R C)$ et d'entretien $(R E)$ :

$$
F P=A P+T r+R C+R E
$$

\section{Accroissement pondéral}

Le cumul moyen des variations de diamètre de chaque traitement a été calculé sur $24 \mathrm{~h}$ à partir de la moyenne des 5 mesures micromorphométriques sur pêches. Pour transformer cet accroissement moyen en gain pondéral, nous avons établi la relation statistique propre à ce cultivar entre la masse de matière fraîche $(M F)$ et le diamètre interjugal $(D)$ :

$$
M F \mathrm{~g}=\mathfrak{f}(D \mathrm{~mm})
$$

Pour établir cette relation nous avons régulièrement prélevé des échantillons de pêches de ce cultivar, tout au long de la période d'expérimentation, dans un verger situé à une centaine de mètres de notre dispositif.

\section{Transpiration}

La transpiration par unité de surface de la pêche dépend des conditions climatiques et de la perméabilité surfacique du fruit. La transpiration des pêches en place a été évaluée par pesées régulières de lots de pêches cueillies, suspendues par un fil, à $50 \mathrm{~cm}$ du sol. Ce dispositif a été décidé sur des bases pratiques (éviter l'effet thermique d'un sol trop proche, ne pas présenter trop de prise au vent) ; il ne prétendait pas simuler les conditions microclimatiques dans les arbres de notre dispositif expérimental. II avait pour objectif d'explorer la variabilité de la transpiration surfacique des pêches dans des conditions climatiques contrastées. Au cours de la dernière quinzaine de juin 
une dizaine d'essais différents ont été conduits. Chaque essai a consisté à suivre (pendant 1 à $3 \mathrm{~h}$ ), à des moments différents de la journée, la variation de masse des pêches, à un pas de temps qui a varié selon les essais (5 à $15 \mathrm{~min}$ ). Chaque essai a porté sur un échantillon allant jusqu'à 20 fruits, prélevés au même moment dans un verger en tentant de respecter la variabilité des calibres présents. Après cueillette et paraffinage de la cicatrice pédonculaire, les fruits étaient pesés puis immédiatement suspendus au dispositif installé à proximité du laboratoire, sur un gazon, sans risque d'ombre portée. Les données climatiques horaires ont été enregistrées par la station météorologique de notre centre de recherches. Nous avons établi sur 699 mesures une relation statistique, relation [3], reliant la transpiration horaire au rayonnement global, à la masse de matière fraîche du fruit et à sa surface (calculée en assimilant la pêche à une sphère). La transpiration journalière est obtenue par intégration sur $24 \mathrm{~h}$ des transpirations horaires.

\section{Respiration}

Les pertes, en bilan pondéral pour le fruit, sont limitées à la seule perte des atomes de carbone sous forme de $\mathrm{CO}_{2}$ : l'oxydation des sucres libère de l'eau qui reste dans le fruit et l'oxygène nécessaire à la réaction vient des échanges avec l'atmosphère. Les pertes par respiration ont été calculées grâce aux relations établies pour la pêche par Dejong et Goudrian (1989). Ces pertes par respiration sont négligeables par rapport à la croissance pondérale et à la transpiration : en effet, au cours de la période d'étude, le cumul de ces pertes correspond, au maximum, à $0,4 \%$ du cumul des flux pédonculaires (tableau I).

Le flux pédonculaire quotidien, $F P j$, est calculé à partir des relations [1], [2] et [3].

\section{Calcul des flux pédonculaires à l'échelle horaire}

Sur la durée de l'expérience nous avons choisi une période (18 au 24 juin) pour analyser l'évolution nyc- thémérale des flux pédonculaires suivant la disponibilité en eau, pendant la dernière phase de restriction. Au pas horaire, nous avons fait l'hypothèse que les mesures simultanées des microvariations de diamètres de fruits non détachés de l'arbre (arbre témoin et arbre stressé) et d'un fruit détaché laissé dans l'arbre (dans les conditions des autres fruits) permettaient d'évaluer les variations liées à la transpiration et de calculer les flux pédonculaires des 2 traitements. Lang (1990) a utilisé cette méthode sur des pommes en mesurant un fruit détaché pour déduire de sa perte de diamètre la transpiration des pommes en place.

On peut écrire les relations suivantes en ce qui concerne les variations horaires de masse de matière fraîche d'un fruit non détaché $\left(d M F_{1}\right)$ et d'un fruit détaché (dMF2) :

$$
\begin{gathered}
d M F_{1}=F P_{h}-T r_{h} \\
d M F_{2}=T r_{h}
\end{gathered}
$$

avec $F P_{h}=$ accroissement dû au flux pédonculaire ; $T r_{h}=$ perte due à la transpiration, la respiration étant considérée comme négligeable; d'où la relation :

$$
F P_{h}=d M F_{1}-d M F_{2}
$$

où $d M F_{1}$ et $d M F_{2}$ sont obtenus à partir des masses du fruit en place et du fruit cueilli, calculées par la relation [2] appliquée à leurs diamètres à $1 \mathrm{~h}$ d'intervalle. II s'agit d'estimations faites à partir des mesures réalisées sur 3 pêches de calibres voisins, 1 sur chaque traitement (pêche témoin et pêche stressée) et 1 pêche récemment cueillie. La validité de cette estimation repose sur 2 hypothèses :

- les effets de dilatation thermique sont négligeables, que le fruit soit en place ou détaché : cela est acceptable au pas horaire, puisque les écarts de température sont faibles, que les fruits sont d'un calibre voisin et qu'ils sont exposés aux mêmes conditions microclimatiques ;

- le fruit détaché transpire, au moins quelques jours, comme s'il était en place non cueilli ; la vérification a été faite par Jones et Higgs (1982) sur pommes et par Lang et Thorpe (1989) sur baies de raisin.

\begin{tabular}{|c|c|c|c|}
\hline $\begin{array}{c}\text { Flux } \\
\text { pédonculaire }\end{array}$ & Transpiration & Respiration & $\begin{array}{c}\text { Accroissement } \\
\text { pondéral }\end{array}$ \\
\hline 482 & $329(68,3)$ & $2(0,4)$ & $151(31,3)$ \\
\hline 317 & $223(70,3)$ & $1(0,3)$ & $93(29,3)$ \\
\hline
\end{tabular}

Tableau I. Bilan des flux massiques (g) concernant la pêche Dixired dans sa dernière phase de croissance (31 j) suivant la disponibilité en eau.

Entre parenthèses, expression (en \%) du flux pédonculaire entrant. Les valeurs de ce tableau ont été obtenues par cumul des flux journaliers calculés, présentés sur la figure 3 . 


\section{RÉSULTATS}

\section{Effet global du stress hydrique sur la croissance de la pêche}

La figure 1 présente les variations cumulées observées, pour chaque traitement, du diamètre du tronc et d'une pêche parmi les 5 pêches suivies par micromorphométrie sur ce traitement, choisie car elle était la plus représentative de l'accroissement diamétral moyen $\left(A D M_{5}\right)$ de cet échantillon de 5 pêches au cours de la période du 29 mai au 23 juin. La pêche témoin a un accroissement diamétral de $34,78 \mathrm{~mm}\left(A D M_{5}\right.$ témoin $=34,30$; écart type $=4,91)$. La pêche stressée a un accroissement diamétral de 22,55 $\mathrm{mm}\left(A D M_{5}\right.$ stressé $=22,31$; écart type $\left.=6,11\right)$.

Pendant la période initiale, sans restriction, les 2 arbres observés se comportent de façon identique. À l'arrêt de l'irrigation (matin du 8/06) l'arbre stressé se démarque rapidement : le tronc passe en décroissance, la croissance de la pêche est arrêtée. Les maxima de contraction diurne (MCD) du tronc et du fruit de l'arbre stressé augmentent considérablement par rapport à l'arbre témoin. Le 10 juin la valeur du MCD du tronc de l'arbre stressé est 4 fois plus forte que celle observée sur cet arbre avant l'arrêt de l'irrigation. La remise en irrigation pendant quelques jours rétablit pour l'arbre stressé un taux de croissance du fruit identique à celui de l'arbre témoin mais sans permettre de rattrapage. Le tronc se réhydrate partiellement mais ne retrouve pas son diamètre d'avant stress. Les MCD des 2 arbres redeviennent voisins.

Pendant la deuxième période de restriction, les mêmes phénomènes se retrouvent, mais moins accentués : sur l'arbre stressé le diamètre du fruit observé s'écarte de plus en plus de celui du fruit observé sur l'arbre témoin ; sur l'arbre stressé les MCD du tronc sont systématiquement plus forts que ceux de l'arbre témoin. En fin de période, le fruit de l'arbre témoin a dépassé son stade de maturité, puisqu'il atteint un maximum de diamètre le 23 juin pour décroître ensuite, ce qui n'est pas le cas du fruit de l'arbre stressé. À partir du premier arrêt de l'irrigation (8 juin) jusqu'à la fin de l'expérience, la moyenne des MCD du tronc du pêcher stressé a été 3 fois plus forte que la valeur de cette moyenne sur ce tronc avant toute restriction de l'alimentation en eau.

Le caractère significatif, à partir de la deuxième période de restriction, de l'effet du stress hydrique sur la croissance est confirmé pour l'ensemble des arbres étudiés grâce aux données du

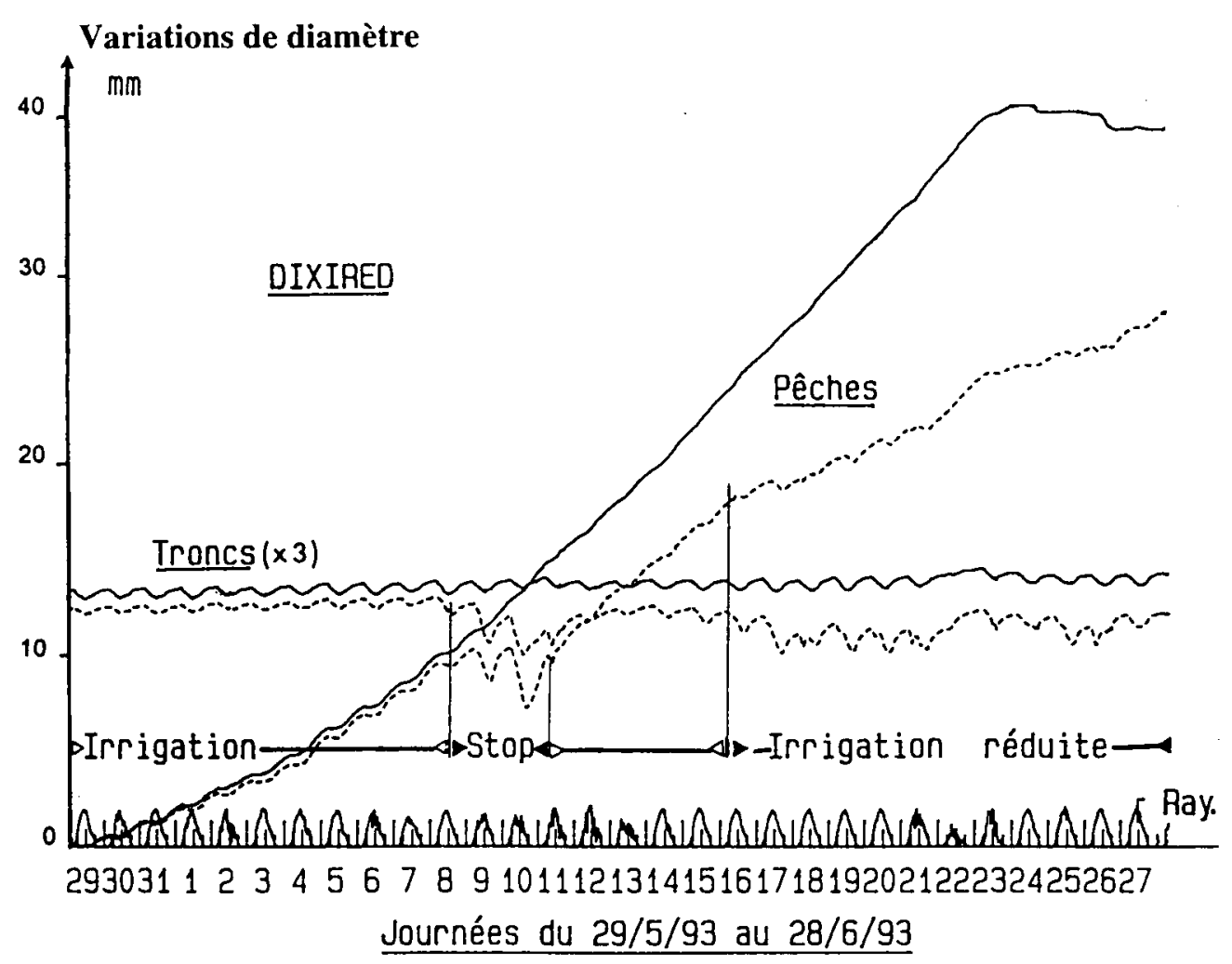

Fig 1. Variations du diamètre des troncs et de pêches, du cultivar Dixired pendant la phase III de croissance des fruits, suivant la disponibilité en eau. - arbre avec irrigation non limitante ; -... arbre avec irrigation limitante. Les ordonnées à l'origine sont arbitraires et n'ont aucune relation avec le diamètre réel des organes mesurés au début de l'expérience. Les variations concernant les troncs ont été multipliées par 3. 


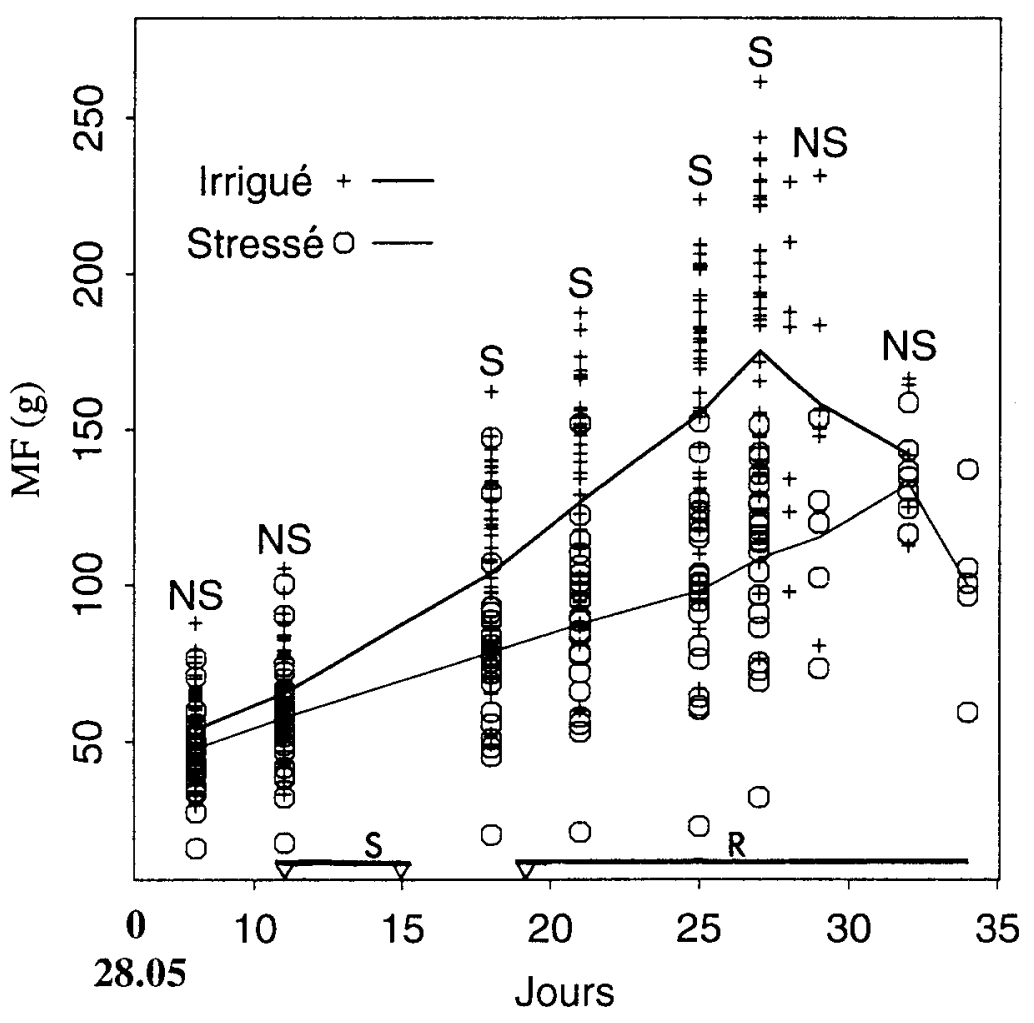

Fig 2. Croissance massique des pêches suivant la disponibilité en eau : valeurs individuelles et moyennes sur l'ensemble des arbres en traitement. La masse de matière fraîche (MF) a été calculée grâce à la relation [2] (cf texte) à partir de la valeur du diamètre. Irrigué : arbres avec irrigation non limitante ; Stressé : arbres avec irrigation limitante. Les 2 périodes de stress en eau sont repérées sur l'axe des abcisses : $S$ : arrêt de l'irrigation; $\mathrm{R}$ : réduction de l'irrigation. $\mathrm{S}$ : différence significative ; NS : différence non significative $P<0,05$ (analyse de variance) suivi manuel de la croissance de tous les fruits (fig 2).

\section{Effet du stress hydrique sur les flux affectant la pêche}

\section{Variations journalières des flux au cours des dernières semaines de croissance}

L'échantillon de pêches Dixired de différents calibres que nous avons prélevé au cours de cet essai nous a permis d'établir la relation suivante (établie sur une population de 242 pêches du même cultivar) entre le diamètre inter-jugal $(D$, en $\mathrm{mm}$ ) et la masse de matière fraîche de la pêche $(M F$, en $g)$ :

$$
M F=0,00325 \times(D)^{2,55} r=0,99 ; P<0,001 \text { [2] }
$$

La mesure des transpirations des fruits cueillis a permis d'établir la relation [3], permettant de calculer, à l'échelle de l'heure et du fruit individuel, la transpiration $\left(T r_{h}\right.$ en g par heure et par pêche) :

$$
\begin{gathered}
T r_{h}=T r_{\max } \times[1-\exp (-0,005 \times M F \times R G)] \times S \\
r=0,55 ; P<0,001
\end{gathered}
$$

avec $T r_{\text {max }}$ : transpiration surfacique maximale $=$ $0,012 \mathrm{~g} \mathrm{x} \mathrm{h}^{-1} \times \mathrm{cm}^{-2}, R G$ : rayonnement global en $10^{6}$ joules $\times \mathrm{M}^{-2} \times \mathrm{h}^{-1}$ et $S$ : surface du fruit en $\mathrm{cm}^{2}$.

La figure 3 montre l'évolution temporelle des flux pédonculaires massiques (calculés à partir des relations [1], [2] et [3]) ainsi que les parts utilisées pour la croissance en masse du fruit et la transpiration. La croissance en masse de la pêche de chaque traitement a été calculée à partir de l'évolution de la masse moyenne obtenue en appliquant la relation [2] aux diamètres observés chaque jour sur les 5 pêches suivies constamment par micromorphométrie. La validité de cette représentation repose sur le réalisme de l'estimation de la transpiration qui sera discuté ultérieurement. Chez l'arbre témoin, le flux pédonculaire augmente de 5 à $35 \mathrm{~g} / \mathrm{jour}$ au cours de la période. La transpiration augmente aussi en valeur absolue mais diminue lors d'une période de temps couvert. Pour l'arbre stressé, l'arrêt de l'irrigation diminue rapidement la croissance pondérale et, en conséquence, le flux pédonculaire de façon parallèle. La restriction pratiquée à partir du 16 juin diminue aussi le flux de l'arbre stressé mais permet une croissance de l'ordre de 0 à $6 \mathrm{~g} /$ jour pour des valeurs de 5 à $11 \mathrm{~g} / \mathrm{jour}$ chez l'arbre témoin. Sur la durée de l'essai, l'accroissement pondéral moyen des pêches du traitement stressé a été réduit à $62 \%$ de celui du témoin (tableau I). Les fortes valeurs de croissance observées au moment de la pluie $(13 \mathrm{~mm}$ le 22 juin, $26^{\mathrm{e}}$ jour de la figure 3 ) relèvent proba- 

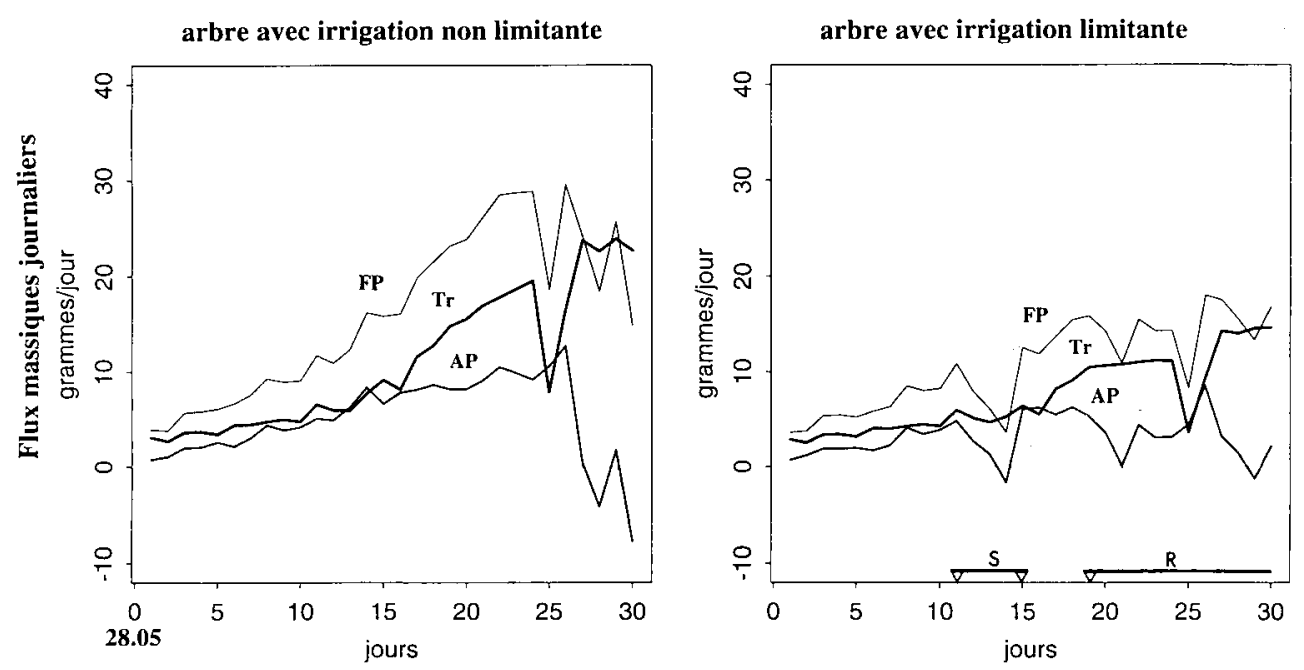

Fig 3. Évolution des différents flux massiques journaliers concernant la pêche Dixired dans sa dernière phase de croissance selon la disponibilité en eau. L'accroissement pondéral (AP) et la transpiration (TR) sont calculés à partir des données mesurées (diamètre des pêches, rayonnement global) et de diverses formules ( $f$ texte). Le flux pédonculaire (FP) est calculé sur la base du bouclage de bilan sur ces paramètres. Les 2 périodes de stress en eau sont repérées sur l'axe des abcisses : $S$, arrêt de l'irrigation ; R, réduction de l'irrigation.

blement d'une pénétration directe par humectation des fruits.

La transpiration propre des pêches représente une proportion du flux pédonculaire massique, en moyenne, voisine de $70 \%$ au cours de cette phase (tableau I). Cela représente pour un fruit une perte d'eau d'environ $20 \mathrm{~g}$ par jour à l'approche de la maturité.

Le maximum de contraction diurne du tronc, indicateur de contrainte hydrique

La figure 1 montrait, chez l'arbre stressé, une liaison négative visible entre le MCD du tronc et le taux de croissance du fruit. Elle peut être exprimée par une relation linéaire positive et très significative $(r=0,9 ; P<0,001)$ entre le rapport des flux pédonculaires journaliers $\left(F p j_{\text {stressé }}\right.$ $F P j_{\text {témoin }}$ ) et le rapport des maxima de contraction diurne $\left(M C D_{\text {témoin }} / M C D_{\text {stressé }}\right)$. Autrement dit, la croissance de la pêche de l'arbre stressé est d'autant moins forte par rapport à celle de la pêche de l'arbre témoin que le $M C D$ du tronc du pêcher stressé est plus fort par rapport à celui du témoin.

\section{Variations au cours de la journée des flux pédonculaires massiques de la pêche}

La figure 4 permet de comparer, à l'échelle horaire et sur la période du 18 au 22 juin, l'évolution des flux pédonculaires de pêches, sur les mêmes arbres que précédemment avec ou sans contrainte hydrique. L'arbre témoin reçoit $12 \mathrm{~h}$ d'irrigation continue pendant chaque phase diurne. L'arbre stressé est irrigué $3 \mathrm{~h}$ de suite en début de matinée. Le dernier jour (22 juin) la diminution simultanée de la variabilité des flux durant le nycthémère et de leurs valeurs moyennes à l'échelle journalière est à relier au temps très couvert et pluvieux.

\section{Comportement sans restriction hydrique, pêche de l'arbre témoin}

Il existe une périodicité nycthémérale très nette dans l'évolution de la transpiration et donc du flux pédonculaire. Ce dernier présente un minimum, nul ou négatif, vers $7 \mathrm{~h}$ (TU) et un maximum à $16 \mathrm{~h}$. Globalement le flux pédonculaire, sans restriction hydrique, et la transpiration paraissent donc en phase avec la croissance diurne de la demande climatique, leur intensité diminuant ensuite régulièrement jusqu'au lever du jour suivant.

\section{Comportement avec restriction hydrique, pêche de l'arbre stressé}

Le fruit de l'arbre stressé montre un flux pédonculaire qui diminue dès l'arrêt de l'irrigation jusqu'au moment du maximum de demande climatique dans l'après-midi. La transpiration n'est plus compensée par le flux pédonculaire, le fruit stressé diminue de diamètre et de masse. Quand la demande climatique diminue, le flux pédonculaire augmente pour rejoindre le niveau de flux du fruit témoin, vers $19 \mathrm{~h}$ TU. Dès que la disponibilité 


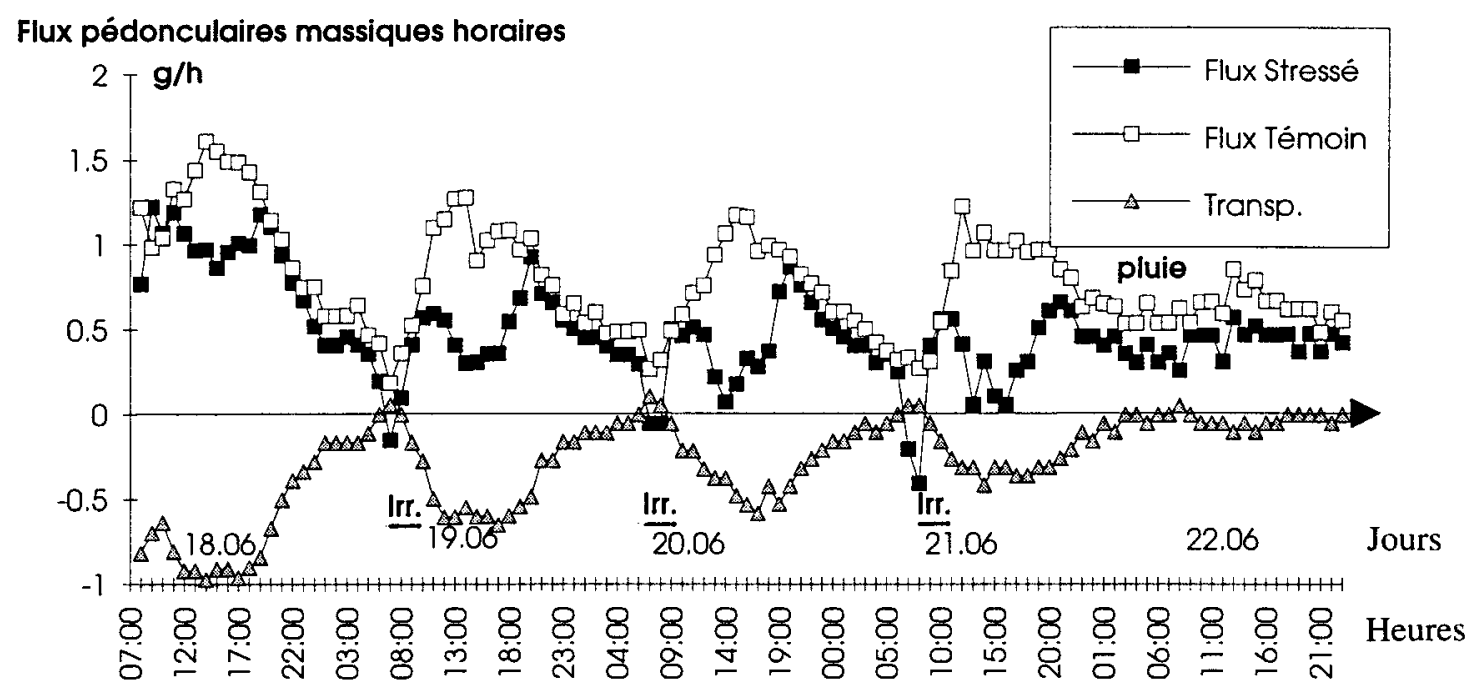

Fig 4. Évolution nycthémérale des flux massiques pédonculaires horaires de pêches de 2 pêchers «Dixired", l'un soumis à une restriction en eau, l'autre non. Valeurs calculées à partir de données de diamètre acquises, pour chaque arbre, sur une pêche non détachées, et sur une seule pêche détachée, selon la méthode décrite dans Matériel et méthodes. Flux stressé : flux correspondant à l'arbre avec une irrigation limitante ; flux témoin : flux correspondant à l'arbre avec une irrigation non limitante ; Transp : transpiration calculée sur la base des variations de diamètre de la pêche détachée. Le segment horizontal placé sous les symboles «Irr» matérialise le moment et la durée $(3 \mathrm{~h})$ de la période d'irrigation accordée à l'arbre stressé.

hydrique est réduite, pendant la phase éclairée du nycthémère, le flux pédonculaire évolue à contre-phase de la demande climatique, comportement qui est inverse de celui du témoin. De l'arrêt de l'irrigation de l'arbre stressé jusqu'à la fin de l'après-midi, les trajectoires de flux des 2 traitements sont complètement en opposition. La surface du parallélogramme délimité par ces trajectoires donne une image quantitative du déficit de flux imposé par une restriction de la disponibilité en eau. Ainsi apparaît nettement l'incidence de l'heure de distribution de l'eau dans une situation où la quantité d'eau disponible est insuffisante à satisfaire à la fois la demande en eau des feuilles et des fruits. Dans une telle situation, si l'objectif est de minimiser le déficit de flux pédonculaire provoqué par le stress hydrique, il vaut mieux irriguer pendant la matinée, au cours de la phase croissante de la demande climatique.

\section{DISCUSSION}

\section{Méthodes d'appréciation des flux}

En l'état actuel des connaissances il est très difficile, voire impossible, d'accéder de façon directe aux différents flux qui agissent sur la croissance d'un fruit en place. La relation masse $x$ diamètre est une relation hautement significative, couramment utilisée, mais dont les paramètres sont spécifiques d'une variété et qu'il ne faut pas appli- quer à des fruits accidentellement déformés. Pour estimer la transpiration de fruits en place, Selles (1988) a utilisé in situ une chambre de mesure des échanges gazeux, méthode qui fournit des résultats ponctuels mais qui perd son intérêt lorsqu'il faut évaluer la dynamique de la transpiration d'un fruit en relation avec l'évolution du microclimat dans l'arbre. Au pas horaire nous avions fait l'hypothèse que la mesure des microvariations de diamètre d'un fruit détaché, laissé dans l'arbre (dans les conditions des autres fruits), permettrait d'évaluer les variations liées à la transpiration, nécessaires au calcul du flux pédonculaire des fruits en place. La comparaison des flux pédonculaires horaires (fig 4) s'est faite en suivant $5 \mathrm{j}$ de suite la même pêche, cueillie le matin du 18 juin. II est certain que, dans les mêmes conditions climatiques et par rapport à une pêche en place, la pêche cueillie risque de perdre de moins en moins d'eau à mesure qu'elle se dessèche. Les valeurs des transpirations estimées (et des flux pédonculaires) seraient donc d'autant plus sous-estimées que l'on s'éloigne du matin du 18 juin. Ce phénomène est visible sur la figure 4 où la transpiration et, par conséquent les flux pédonculaires maximaux, diminuent régulièrement du 18 au 20 juin alors que les conditions climatiques de ces 3 journées étaient pratiquement identiques ( $c f$ la courbe du rayonnement de la figure 1). Entre le 18 juin à $13 \mathrm{~h}$ et le 20 juin à $13 \mathrm{~h}$ le flux horaire maximal (fruit de l'arbre témoin) a diminué de $30 \%$ pour un cumul d'approximativement $20 \mathrm{~h}$ d'ensoleillement. Si l'on 
veut ne pas dépasser $5 \%$ de sous-estimation de la transpiration réelle, l'utilisation d'une pêche cueillie pour apprécier la transpiration de pêches en places ne devrait donc pas dépasser $3 \mathrm{~h}$ pendant une phase diurne.

Nous avons évalué la transpiration d'une pêche soit par la relation 3 tout au long de la période d'expérimentation, soit à partir des variations horaires de diamètre d'une pêche détachée pour la période du 18 au 22 juin. II est alors possible de comparer les transpirations journalières obtenues par les 2 méthodes au cours des 3 j $(19,20$ et 21 juin) pour lesquels nous avons une continuité des observations sur la pêche cueillie. Le calcul par la relation [3] (pêche de l'arbre témoin) conduit à des valeurs toujours plus fortes (136\% le $19,151 \%$ le $20,157 \%$ le 21 juin) que les estimations à partir du fruit détaché ; l'écart augmente avec l'áge du fruit détaché, qui le 19 juin au matin avait déjà été cueilli depuis $24 \mathrm{~h}$. Cette comparaison confirme les conclusions tirées précédemment de l'analyse de l'évolution de la transpiration visible sur la figure 4. L'hypothèse de représentativité d'un fruit cueilli par rapport à la transpiration d'un fruit en place serait donc acceptable d'autant plus longtemps qu'il s'agit d'une espèce dont la transpiration surfacique des fruits est plus faible. Mais malgré la sous-estimation de la transpiration et des flux pédonculaires, la comparaison des évolutions des flux de la pêche de l'arbre témoin et de la pêche de l'arbre stressé, présentées sur la figure 4 , reste valable puisque ces évolutions ont toutes les 2 été calculées relativement à la transpiration de la même pêche détachée. La sous-estimation de la transpiration d'une pêche par une utilisation trop longue du même fruit détaché est certaine, mais l'application de la relation [3] a dû surestimer la transpiration (dans une proportion inconnue) car notre dispositif d'exposition des fruits cueillis aux variations climatiques imposait des conditions certainement plus sévères que celles subies par des pêches en place dans un arbre.

\section{Flux pédonculaire}

Ce que nous appelons flux pédonculaire est en fait le bilan des flux xylémien ou phloémien. Les valeurs du flux pédonculaire, faibles, voire négatives, au lever du jour, doivent correspondre à une inversion de flux qui pourrait être due à la décroissance brutale du potentiel hydrique dans les tiges résultant d'un déphasage momentanément intense entre l'absorption racinaire et la transpiration foliaire au moment de l'ouverture stomatique. L'inversion de flux est une hypothèse qui a été faite par Lang (1990) chez le pommier. En fin de croissance des pêches, le phénomène d'inversion de flux affecte seulement le fruit stressé. L'interprétation suivante peut être proposée : la diminution des potentiels osmotiques des fruits en rapport avec leur enrichissement en sucres n'autorise de sortie d'eau que dans le cas de potentiels xylémiens très négatifs. Dans le cas d'une insuffisance de la disponibilité en eau, la concurrence feuilles-fruits se traduit par une baisse du flux pédonculaire des fruits tant que la transpiration des feuilles est croissante ; le potentiel hydrique du fruit reste inférieur à celui de la tige, mais la différence s'amenuise à mesure que les feuilles transpirent davantage. Dès que la transpiration des feuilles cesse de croître le phénomène s'inverse.

Contrairement à l'hypothèse de flux constant retenue par Lee (1990) dans sa modélisation du bilan hydrique de la tomate et de la cerise, le flux pédonculaire journalier de la pêche augmente régulièrement : résultat qui a été confirmé récemment par ceux obtenus sur un brugnon $\mathrm{cv}$ Big-Top que nous avons étudié pendant la phase III (du 20 mai au 5 juillet 1994). Le flux pédonculaire passait de 2 à $7 \mathrm{~g} / \mathrm{j}$ pour des fruits dont le poids maximal est environ la moitié celui du cv Dixired. Chez Dixired comme chez BigTop le flux pédonculaire décroît rapidement à l'approche de la maturation. Pour interpréter cet arrêt, il est possible de mettre en cause la différenciation de la couche d'abcission qui, chez le pêcher entre autres espèces, détruit la continuité du système vasculaire au moment de la maturité. Par ailleurs nous avons remarqué que les pêches de l'arbre témoin arrivent à maturité plusieurs jours avant celles de l'arbre stressé ; ce retard de maturité par le déficit hydrique a déjà été signalé sur le raisin par Hardie et Considine (1976).

\section{Compensation de la transpiration}

Chez la pêche, la transpiration évacue de l'ordre de $70 \%$ du flux pédonculaire total entrant dans le fruit pendant la phase finale de grossissement. Pour la pêche, il paraît donc nécessaire qu'une compensation rapide de la transpiration soit possible, ce que permettrait plus facilement la voie xylémienne, en raison de la plus faible viscosité de la sève brute. II est donc possible que l'efficacité du xylème doive se maintenir plus longtemps chez la pêche que pour des espèces telles que la tomate, le raisin et la pomme, qui transpirent peu et chez qui a été prouvée la prédominance finale 
de la voie phloémienne (Ho et al, 1987 ; Lang et al, 1989 ; Jones et Higgs, 1982). Si les voies xylémienne et phloémienne conservent longtemps leur efficacité chez la pêche, il serait très instructif, sous l'angle de la qualité du fruit, d'apprécier la sensibilité relative de leurs flux aux effets d'une contrainte en eau.

\section{Flux de transpiration}

\section{Variabilité dans l'espèce pêcher}

La transpiration surfacique moyenne d'une pêche cv Dixired (témoin bien irrigué), pour la période

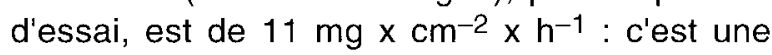
valeur 3 fois plus forte que celle trouvée par Selles (1988) pour une pêche cv Fire-Red et pour une période comparable. Nous avons trouvé dans nos essais sur brugnon Big-Top une transpiration surfacique 2 fois plus faible que pour Dixired (Chaar, 1994). Nobel (1975) trouve sur des pêches (cv Alberta et Golden-Queen) des conductances épidermiques 2 à 3 fois plus faibles que celle mesurée dans les mêmes conditions par Selles sur le cv Fire-Red. Ces fortes différences variétales existent aussi chez le pommier où Jones et Higgs (1982) trouvent des conductances surfaciques variant d'un facteur 1 à 3 sur différentes variétés. La variabilité de la conductance surfacique est certainement très forte chez l'espèce pêcher en raison de la variabilité de la nature de l'épiderme, lisse ou duveteux. Nguyen The et al (1989) ont montré que le réseau des micro-craquelures était très dense et que cette densité, donc la perméabilité surfacique, augmentait avec la taille du fruit (Nguyen The, communication personnelle).

Si la transpiration propre des pêches paraît très forte, elle ne constitue pas nécessairement le moteur de la croissance du fruit. L'observation de la figure 3 à la date du $25^{\mathrm{e}} \mathrm{j}$ fait apparaître que la très forte baisse de la transpiration liée aux conditions climatiques ne modifie pas l'accroissement pondéral. Sur des pêchers dont l'alimentation en eau n'était pas limitante, nous avons aussi fait des essais de blocage de la transpiration de pêches, en place, en les enfermant sous un film plastique transparent sans obtenir de modification significative de leur vitesse de croissance par rapport aux fruits transpirant librement. La transpiration ne paraît donc pas indispensable au grossissement du fruit même si elle représente un flux de sortie quantitativement très important.

\section{Variabilité suivant les espèces}

L'importance relative de la transpiration propre de la pêche distingue très nettement le comportement de ce fruit de celui d'autres espèces. La comparaison des transpirations observées sur pêches cueillies à celles obtenues sur baies de raisin (Lang et Thorpe, 1989), sur tomate (Lee, 1989) et sur pommes (Lang, 1990) indique que la transpiration de la pêche est beaucoup plus forte. Dans le classement des fruits à maturité selon leurs résistances cuticulaires (Nobel, 1975), le raisin et la pomme ont respectivement des résistances 5 fois à $60-200$ fois plus fortes que la pêche. Ces ordres de grandeur, sur pommes, sont confirmés par les mesures de Jones et Higgs (1982).

\section{CONCLUSIONS}

Ce travail nous a permis de préciser, chez la pêche Dixired dans sa phase III de croissance, l'incidence quantitative d'une restriction de la disponibilité en eau sur l'évolution des flux qui déterminent le grossissement des fruits. Toute restriction hydrique subie par l'arbre conduit à une diminution de la croissance des pêches en relation quantitative avec l'augmentation des maxima de contraction diurne des troncs. Le maintien probable, jusqu'à la maturité, d'un flux xylémien important reste à prouver chez la pêche, comme il reste à étudier l'effet d'une restriction en eau sur l'importance relative des flux xylémien et phloémien, qui pourrait expliquer l'augmentation de la teneur en sucres des fruits après souffrance hydrique. La transpiration de la pêche ne paraît pas jouer un rôle moteur indispensable à son grossissement. En revanche, lorsqu'il y a contrainte hydrique et sous l'angle qualitatif, il paraît tout à fait primordial d'apprécier les modifications de teneur en sucre liées au degré de compensation de la transpiration d'eau pure par le jeu relatif des voies xylémienne ou phloémienne.

Nous avons trouvé une relation linéaire très significative entre la diminution, par rapport à une pêche de l'arbre témoin, du flux pédonculaire d'une pêche de l'arbre stressé en eau et l'augmentation du maximum de contraction diurne du tronc de l'arbre stressé par rapport à celui de l'arbre témoin. Cette constatation ouvre des perspectives pratiques quant à la possibilité de construire un modèle de croissance des pêches en fonction de la disponibilité en eau. Le suivi 
micromorphométrique des variations de diamètre des troncs fournirait les MCD qui serviraient de variables d'entrée pour définir l'intensité de la restriction appliquée. Cette modélisation devrait permettre de piloter avec précision des itinéaires de restriction en eau destinés à améliorer la qualité organoleptique des pêches tout en contenant la baisse de calibre dans des limites économiquement acceptables.

\section{REMERCIEMENTS}

Ce travail a été réalisé dans le cadre et avec l'aide financière du programme de l'action incitative programmée "Valorisation et protection des ressources en eau» du secteur Environnement physique et Agronomie de I'Institut national de la recherche agronomique. Nous remercions C Polizzano et B Baculat, station de bioclimatologie d'Avignon, de nous avoir aimablement communiqué les données climatiques horaires.

\section{RÉFÉRENCES}

Bussières $P$ (1994) Water import rate in tomato fruit: a resistance model. Ann Bot (London) 73, 75-82

Chaar H (1994) Influence de l'alimentation hydrique sur la croissance des pêches. Mémoire de DEA, INA-Paris-Grignon, université d'Orsay

Chalmers DJ, Olsson KA, Jones TR (1983) Water relations of peach trees and orchards. In : Water Deficit and Plant Growth, Vol VII, Academic Press, New York

Dejong TM, Goudrian J (1989) Modeling peach fruit growth and carbohydrate requirements: reevaluation of the double-sigmoid growth pattern. J Am Soc Hort Sci 114, 800-804

Delgado M (1994) Pêches et nectarines, relancer la consommation. L'Arboriculture fruitière 475, 36-37

Génard M, Bruchou C, Souty M (1991) Variabilité de la croissance et de la qualité chez la pêche (Prunus persica $L$ Batsch) et liaison entre croissance et qualité. agronomie 11, 829-845

Hardie WJ, Considine JA (1976) Response of grapes to water-deficit stress in particular stages of development. Am J Enol Viticult 27, 2, 55-61

Ho LC, Grange RI, Picken J (1987) An analysis of the accumulation of water and dry matter in tomato fruit. Plant Cell Environ 10, 157-162
Huquet JG (1985) Appréciation de l'état hydrique d'une plante à partir des variations micrométriques de la dimension des fruits ou des tiges au cours de la journée. agronomie 5, 733-741

Huguet JG, Li SH, Lorendeau JY, Pelloux G (1992) Specific micromorphometric reactions to water stress and irrigation scheduling automation. $J$ Hort Sci $67,631-640$

Jones HG, Higgs KH (1982) Surface conductance and water balance of developing apple fruits. J Exp Bot $33,67-77$

Johnson RW, Dixon MA, Lee DR (1992) Water relations of the tomato during fruit growth. Plant Cell Environ 15, 947-953

Lang A (1990) Xylem, phloem and transpiration flows in developing apple (Malus pumilla Mill) fruits. J Exp Bot 41, 645-651

Lang A, Thorpe R (1989) Xylem, phloem and transpiration flows in a grape: application of a technique for measuring the volume of attached fruits to high resolution using Achimedes' principle. J Exp Bot 40, 1069-1078

Lee DR (1990) A unidirectional water flux model of fruit growth. Can J Bot 68, 1286-1290

Lee DR, Dixon MA, Johnson RW (1989) Simultaneous measurements of tomato fruit and stem water potential using in situ stem hygrometers. Can J Bot 67, 2353-2355

Li SH, Huguet JG (1989) Production, qualité des fruits et croissance de pêchers soumis à différents régimes d'alimentation hydrique. Fruits 44, 225232

Li GH, Huguet JG, Schoch PG (1989) Response of peach tree growth and cropping to soil water deficit at various stages of fruits development. $J$ Hort $\mathrm{Sci}$ 64, 541-552

Nguyen-The C, Hugheney R, Arnoux M (1989) Contribution à l'étude des voies de pénétration fongique des nectarines, Monilia laxa et Rhizopus stoIonifer. agronomie 9, 271-276

Nobel PS (1975) Effective thickness and resistance of the air boundary layer adjacent to spherical plants parts. J Exp Bot 26, 120-130

Selles G (1988) Effet de la contrainte hydrique sur la variation journalière du diamètre des troncs et des fruits, application au déclenchement de l'irrigation du pêcher. Thèse doct ingénieur, ENSA Montpellier

Simmoneau T, Habib R, Goutouly JP, Huguet JG (1993) Diurnal changes in stem diameter depend upon variations in water content. Direct evidence in peach trees. J Exp Bot 44, 615-621 